\title{
Evolução temporal de compostos fenólicos e atividade antioxidante de vinhos tintos brasileiros
}

Mariana Dullius

mdi@hotmail.com

Universidade Federal do Rio Grande do Sul, Instituto de Ciência e Tecnologia de Alimentos, Departamento de Ciências dos Alimentos, Porto Alegre, Rio Grande do Sul, Brasil

\section{Carlos Henrique Pagno}

cpagno@gmail.com

Universidade Federal do Rio Grande do Sul, Instituto de Ciência e Tecnologia de Alimentos, Departamento de Ciências dos Alimentos, Porto Alegre, Rio Grande do Sul, Brasil

\section{Vitor Manfroi}

manfroi@ufrgs.br

Universidade Federal do Rio Grande do Sul,

Instituto de Ciência e Tecnologia de Alimentos,

Departamento de Ciências dos Alimentos, Porto

Alegre, Rio Grande do Sul, Brasil

\section{Maria Goreti Rodrigues Vale}

mgrvale@ufrgs.br

Universidade Federal do Rio Grande do Sul, Departamento de Química, Porto Alegre, Rio Grande do Sul, Brasil

\section{Plinho Francisco Hertz}

plinho@ufrgs.br

Universidade Federal do Rio Grande do Sul,

Instituto de Ciência e Tecnologia de Alimentos,

Departamento de Ciências dos Alimentos, Porto

Alegre, Rio Grande do Sul, Brasil

\section{Alessandro de Oliveira Rios}

alessandro.rios@ufrgs.br

Universidade Federal do Rio Grande do Sul,

nstituto de Ciência e Tecnologia de Alimentos,

Departamento de Ciências dos Alimentos, Porto

Alegre, Rio Grande do Sul, Brasil

\author{
RESUMO
}

Com base no estudo de 90 amostras de vinhos provenientes de sete diferentes regiões brasileiras, o índice de polifenóis totais e a atividade antioxidante apresentaram evolução distinta durante as 55 semanas de armazenamento. As variáveis estudadas apresentaram correlações fortes e muito fortes no decorrer de 55 semanas. A região do Vale do São Francisco exibiu valores mais elevados de polifenóis em todos os tempos analisados, e maior atividade antioxidante até 45 semanas. A região Oeste do Paraná apresentou os mais baixos resultados para ambos os parâmetros em todos os tempos avaliados. A evolução temporal de compostos fenólicos e da atividade antioxidante mostrou que as diferenças entre vinhos procedentes de regiões de origem distintas diminuem com o decorrer do armazenamento. Características aportadas por variáveis meteorológicas podem ter favorecido as diferenças iniciais, enquanto que ao final de 55 semanas as condições de estocagem aproximaram ao máximo os grupos.
\end{abstract}

PALAVRAS-CHAVE: polifenóis; atividade antioxidante; caracterização vitivinícola; terroir brasileiro. 
INTRODUÇÃO

Devido à extensão continental do Brasil, atualmente podem ser descritas diferentes regiões de cultivo para a videira, das quais se esperam distintas expressões varietais. Giovaninni e Manfroi (2009) definiram cinco diferentes terroirs para o Estado do Rio Grande do Sul (Serra Gaúcha, Serra do Sudeste ou Alto Camaquã, Campanha Gaúcha, Campos de Cima da Serra e Costa do Guaíba), dois para o Estado de Santa Catarina (Vale do Rio do Peixe e Serra Catarinense) e um para o conjunto que engloba os Estados da Bahia, Pernambuco e Minas Gerais, denominado Vale do São Francisco. De acordo com estes autores, em cada terroir brasileiro serão encontradas particularidades regionais e ambientais, que influenciarão na qualidade da uva, matéria prima base para a obtenção de vinhos. Somando-se as particularidades de clones às características das variedades e à adaptabilidade das cepas às diferentes condições edafoclimáticas, geram-se produtos viticolamente distintos. Combinando-se os fatores citados às peculiaridades enológicas possíveis de serem adotadas na elaboração dos vinhos, existe uma enorme diversidade de produtos. Dessa forma, o estabelecimento de áreas ambientalmente semelhantes, com a adoção de práticas e métodos de cultivos próprios, visa definir métodos legais para a obtenção de produtos típicos e pode atuar na fiscalização de Denominações de Origem Controlada (DOC's) (KRAMER, 2007).

Diversas propriedades dos vinhos podem ser usadas para a sua caracterização, entre as quais, o seu conteúdo fenólico e as suas capacidades em neutralizar moléculas de Espécies Reativas de Oxigênio (Reactive Oxigen Species - ROS). A Atividade Antioxidante (AOX) geralmente é medida através de métodos em que os compostos presentes nos vinhos neutralizam soluções orgânicas contendo espécies reativas de oxigênio (RE et al., 1999). Por sua vez a concentração de polifenóis pode ser determinada pelo Índice de Polifenóis Totais (IPT) (MAMEDE e PASTORE, 2004) e, devido à constituição variável destes compostos no fruto, há grandes variações entre os potenciais antioxidantes dos vinhos.

Uma série de fatores (enzimáticos e não enzimáticos) desenvolvidos pela planta como forma de responder ao estresse oxidativo determinam a síntese dos polifenóis (VULETA et al., 2010). Entre os mecanismos não enzimáticos, encontram-se substâncias como ascorbato, glutationa e tocoferol além de diversos compostos carotenóides e polifenólicos (SCANDALIOS, 1993). Muitas vezes, esses mecanismos são ativados conjuntamente para reforçarem as defesas da planta (VULETA et al., 2010).

Landrault et al. (2001) descrevem correlações entre o conteúdo de compostos fenólicos e a atividade antioxidante de vinhos. Entretanto, o maior conteúdo destas substâncias fenólicas não implica, necessariamente, em maior atividade antioxidante (RIVERO-PÉREZ, MUÑÍZ, GONZÁLEZ-SANJOSÉ, 2007). A atividade antioxidante de vinhos parece estar mais relacionada ao tipo de compostos fenólicos presentes (RICE-EVANS e MILLER, 1996). Nesse sentido, flavonoides, proantocianidinas e antocianinas são os que apresentam maior potencial antioxidante (FRANKEL, WATERHOUSE e TEISSEDRE, 1995; RICE-EVANS e MILLER, 1996). 
(também denominadas de taninos condensados ou flavan-3-ois) - e os nãoflavonoides, onde os estilbenos constituem os principais representantes do grupo (KENNEDY, MATTHEWS E WATERHOUSE, 2002).

Entre os flavonóis mais recorrentes em vinhos, encontram-se a quercetina e a miricetina (MATTIVI et al., 2006). O 3,5,4'- trihidróxi-estilbeno, ou resveratrol, é considerado o estilbeno mais bioativo em videiras. Apresenta-se sob a forma de dois isômeros ópticos, o cis-resveratrol e o trans-resveratrol. O trans-resveratrol é o único que apresenta atividade biológica, sendo atribuído a ele diversos benefícios à saúde humana (BAVARESCO et al., 2008).

As videiras cultivadas em áreas de elevada altitude, onde a radiação ultravioleta (UV) e a concentração de ácido abcísico (ABA) são mais intensas, podem sintetizar maiores quantidades de resveratrol (CELOTTI et al., 1996, BERLI, 2011) e de antocianinas (MATEUS et al., 2001). A irradiação pela luz UV-B parece estar associada com o aumento na concentração da enzima responsável pela biossíntese dos flavonóides (BERLI, 2011), sendo que estes protegem o material genético das plantas contra danos provocados pelos raios ultravioletas. (KOLOUCHOVÁ-HANZLÍKOVÁ et al., 2004).

Segundo Kramer (2007), não haveria maturação do vinho se não houvesse degradação de compostos polifenólicos, pois é justamente devido à extensa polimerização dos compostos antioxidantes que a dureza e a adstringência do vinho são perdidas. Vinhos de maior idade apresentam avançada degradação de compostos polifenólicos, com predominância, em termos de tonalidade, do amarelo sobre o vermelho (KRAMER, 2007). Além da importância na evolução dos vinhos, os compostos fenólicos, devido a sua propriedade antioxidante, são relatados em estudos epidemiológicos pela sua ação protetora aos sistemas biológicos (KLATSKY, ARMSTRONG e FRIEDMAN, 1992; LONGNECKER, 1994). Entre os principais resultados encontrados na literatura, destacam-se trabalhos que sugerem que o vinho tinto apresenta a capacidade de reduzir o risco de mortalidade por doenças coronarianas (MC ELDUFF e DOBSON, 1997); outros que apontam que a ingestão moderada de vinhos aporta uma adequada concentração de compostos fenólicos, os quais podem contribuir para diminuição no risco de isquemias e à prevenção de tumores malignos (BARON-MENGUY et al., 2007); aqueles que destacam o resveratrol pelas suas propriedades de inibição da agregação plaquetária e pela diminuição do risco de formação de coágulos sanguíneos (HINES et al., 2001); e aqueles que atribuem às antocianinas ações anti-inflamatórias, antimicrobianas e antitumorais (WANG, COA e PRIOR, 1997).

Esse trabalho teve como objetivo comparar vinhos tintos de diferentes regiões brasileiras, em termos de índice de polifenóis totais e da atividade antioxidante, além de caracterizar o perfil de regiões com potencial para estabelecimento e consolidação de denominações de origem controlada. 


\section{MATERIAIS E MÉTODOS}

\section{MATERIAIS}

Foram analisadas noventa (90) garrafas de vinhos tintos apresentados na Amostra Nacional de Vinhos de 2009 (ANAVI 2009), classificadas de acordo com a sua localidade ou região de origem pela Empresa Brasileira de Pesquisa Agropecuária (Embrapa Uva e Vinho), todos provenientes da safra de 2008/2009. As amostras foram reagrupadas (Tabela 1), levando em consideração: a Unidade da Federação na qual se situam (RS, BA, $\mathrm{PE}, \mathrm{SC}, \mathrm{PR}$ ) e a região fisiográfica da qual fazem parte: Encosta Superior do Nordeste (ESN); Sertão (Ser); Campanha Gaúcha (Cam); Campos de Cima da Serra (CCS); Planalto Serrano (PSe); e Planalto de Guarapuava (PGu). Dessa nova divisão, resultaram sete grandes grupos: Vale dos Vinhedos (VVi); Serra Gaúcha (SGa); Vale do Rio São Francisco (VSF); Campanha Gaúcha (Cam); Campos de Cima da Serra (CCS); Serra Catarinense (SCa); e Oeste do Paraná (OPR), com 11, 45, 2, 12, 7, 9 e 4 amostras respectivamente.

As amostras foram identificadas por número seguido de uma letra minúscula $(a, b, c, d, e, f)$ que define o grupo, seguindo a sequência descrita anteriormente. A região fisiográfica ESN foi dividida em dois grupos: VVi e SGa, por levar em conta o fato de que a primeira já possui Indicação de Procedência (IP) e pudesse constituir um cluster de amostras com características distintas.

Após a classificação, as amostras foram armazenadas em frascos âmbar de $15 \mathrm{~mL}$ e mantidas ao abrigo da luz em temperatura e umidade relativa ambiente (média de \pm 22 ㄷ / $70 \%$ UR) até as análises.

Tabela 1 - Identificação das amostras por: Unidade da Federação na qual tiveram origem (RS, BA, PE, SC e PR); região fisiográfica a qual pertencem (ESN=Encosta Superior do Nordeste; Ser=Sertão; Cam=Campanha Gaúcha; CCS=Campos de Cima da Serra; PSe=Planalto Serrano; e PGu=Planalto de Guarapuava); região de origem do vinho (VVi=Vale dos Vinhedos; SGa=Serra Gaúcha; VSF=Vale do Rio São Francisco; Cam=Campanha Gaúcha; CCS=Campos de Cima da Serra; $\mathrm{SCa}=$ Serra Catarinense; e $\mathrm{OPR}=$ Oeste do Paraná); e variedade de uva (Merlot / Cabernet sauvignon).

\begin{tabular}{|c|c|c|c|c|}
\hline Amostra & Variedade & Região Fisiográfica & $\begin{array}{l}\text { Reglão de } \\
\text { Origem do } \\
\text { Vinho }\end{array}$ & $\begin{array}{l}\text { Unidade da } \\
\text { Federação }\end{array}$ \\
\hline $1 \mathrm{a}$ & Merlot & ESN & Vvi & RS \\
\hline $2 a$ & Cabernet Sauvignon & ESN & Vvi & RS \\
\hline $3 a$ & Cabernet Sauvignon & ESN & Vvi & RS \\
\hline $4 a$ & Merlot & ESN & Vvi & RS \\
\hline $5 a$ & Merlot & ESN & Vvi & RS \\
\hline $6 a$ & Cabernet Sauvignon & ESN & Vvi & RS \\
\hline $7 a$ & Merlot & ESN & Vvi & RS \\
\hline $8 a$ & Cabernet Sauvignon & ESN & Vvi & RS \\
\hline $9 a$ & Merlot & ESN & Vvi & RS \\
\hline $10 a$ & Merlot & ESN & Vvi & RS \\
\hline $11 a$ & Cabernet Sauvignon & ESN & Vvi & RS \\
\hline $1 \mathrm{~b}$ & Cabernet Sauvignon & ESN & Sga & RS \\
\hline $2 b$ & Cabernet Sauvignon & ESN & Sga & $\begin{array}{c}\mathrm{RS} \\
\text { (continua) }\end{array}$ \\
\hline
\end{tabular}




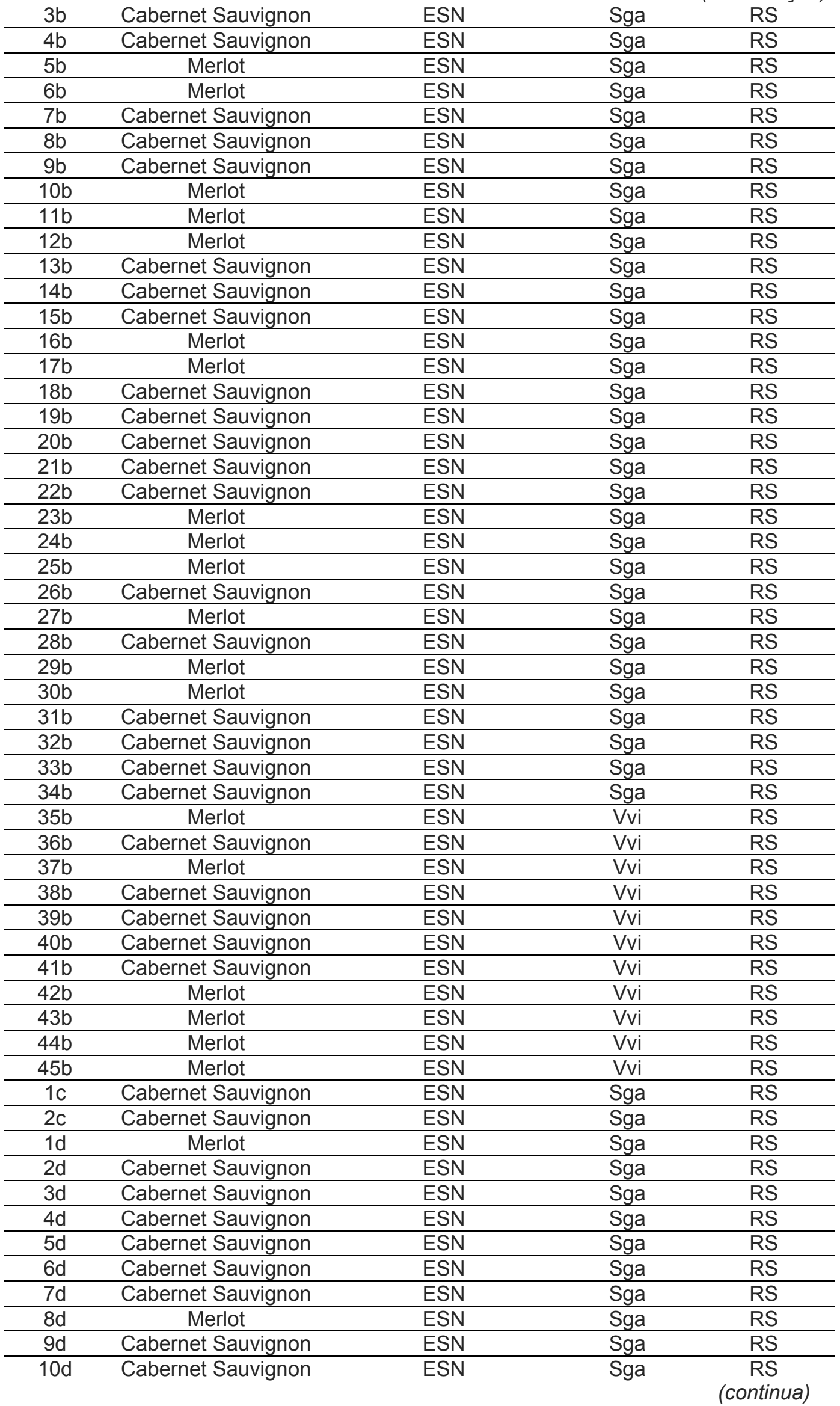




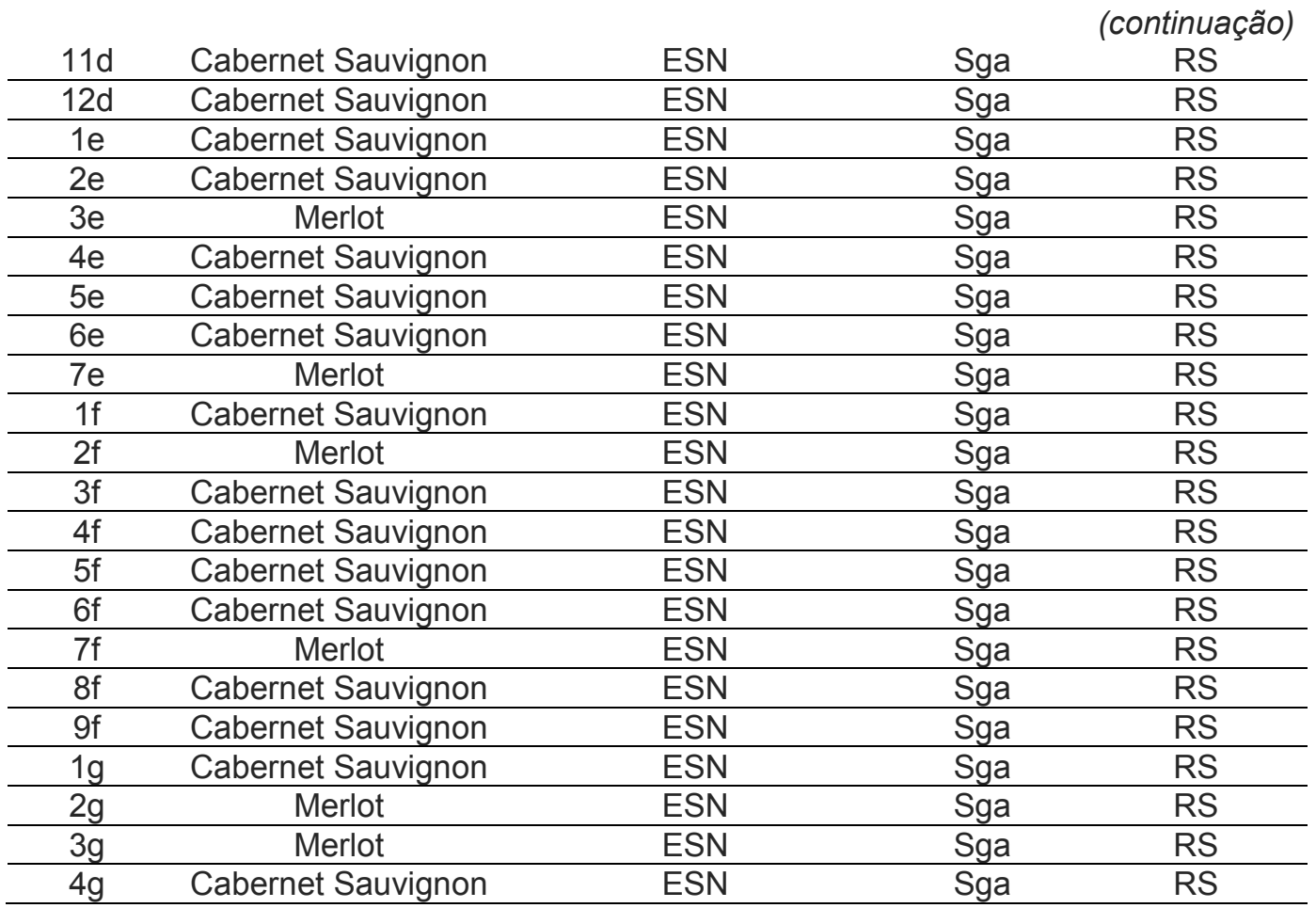

\section{MÉTODOS}

As análises do Índice de Polifenóis Totais (IPT) e Atividade Antioxidante (AOX) foram realizadas em cinco períodos: tempo zero (tZero); 25 semanas (t25); 35 semanas ( $\mathrm{t} 35$ ); 45 semanas ( $\mathrm{t} 45)$; e 55 semanas ( $\mathrm{t} 55$ ) após recebimento das amostras. Em cada tempo de análise, um frasco de $15 \mathrm{~mL}$ de cada amostra era analisado e, na sequência, descartado.

A concentração do IPT dos vinhos foi determinada pelo método colorimétrico Folin Ciocalteau's descrito por SINGLETON, ORTHOFEN e LAMUELARAVENTÓS, (1999). As leituras foram executadas em comprimento de onda de 765 nm no espectrofotômetro UV-Vis-3000 PRO (Amersham-Biosciences). Os reagentes utilizados foram de grau analítico e as reações foram conduzidas em triplicata.

Para quantificação foi empregada uma curva padrão com solução de ácido gálico (Sigma-Aldrich). Os resultados foram expressos em mg.L-1 de ácido gálico, ou em equivalente de ácido gálico (GAE).

A AOX das amostras foi determinada usando o método de neutralização do radical 1,1-difenil-2-picril-hidrazil (DPPH+) (BRAND-WILLIAMS,1995). As leituras foram executadas em espectrofotômetro UV-Vis-3000 PRO (AmershamBiosciences) no comprimento de onda de $515 \mathrm{~nm}$. Os reagentes utilizados foram de grau analítico e as reações foram conduzidas em triplicata. A AOX foi calculada através de curvas de calibração construídas tendo o TROLOX (Sigma-Aldrich) como um antioxidante padrão. Os resultados foram expressos em $\mu \mathrm{M}$ de TROLOX ou em Capacidade Antioxidante Equivalente ao TROLOX (TEAC). 
Os resultados foram submetidos à Análise de Variância (ANOVA) a 95\% de probabilidade estatística. Correlações de Pearson foram aplicadas a um grau de significância de $95 \%$. E diferença entre duas media foi avaliada pelo teste $t$ de student ao nível de $90 \%$ de significância. Os resultados foram discutidos de acordo com: tempo de análise em semanas; evolução temporal dos parâmetros IPT e AOX; região de origem das amostras (grupo); e variedade (Merlot ou Cabernet sauvignon).

\section{RESULTADOS E DISCUSSÃO}

Devido ao fato dos vinhos serem resultado de um processo de síntese e acúmulo de compostos fenólicos que tiveram origem na videira (HORNSEY, 2007), as condições agroclimáticas devem ser consideradas, de forma a compreender as possíveis diferenças existentes entre os grupos, as quais possam ser resultado da combinação do ambiente (clima, solo, planta) com o manejo (práticas agronômicas e enológicas) adotado. Para tanto foram utilizados dados de variáveis meteorológicas fornecidas pelo Centro Estadual de Meteorologia, Fundação Estadual de Pesquisa Agropecuária (CemetRS/FEPAGRO) e pelo Instituto Nacional de Meteorologia (INMET) (Tabela 2).

Dentre as regiões avaliadas, observou-se redução no IPT (Figura 1), ao longo das semanas de armazenamento no laboratório, acompanhada pela perda da AOX (Figura 2). A presença de oxigênio dentro dos frascos contendo o vinho pode ocasionar a degradação de antocianinas (CANO-LÓPEZ et al., 2007; KRAMER, 2007), podendo ocorrer a perda de cor e a formação de copolímeros de tonalidade amarelada, as quais imprimem no vinho uma aparência de envelhecido.

A maior diferença, em termos de valores iniciais e finais de IPT, foi observada nos vinhos provenientes da região VSF, em que as amostras partiram dos mais elevados valores de IPT (5.170 GAE) e AOX (17.125 TEAC) e reduziram até $1804 \mathrm{GAE}$ e $5.498 \mathrm{TEAC}$, respectivamente. As regiões que menos oscilaram, em termos de valores iniciais e finais de IPT, também foram aquelas que apresentaram os valores iniciais mais baixos, como a região OPR (2.104 a 1.008 GAE; 8.397 a 4.346 TEAC) e a região da SGa (2.738 a 1.426 GAE; 9.669 a 5.169 TEAC). Pela ANOVA, pelo menos uma das regiões difere das demais $(p<0,05)$ para IPT e AOX.

A região do VSF apresentou os maiores dados de IPT e AOX. A elevada AOX encontrada em vinhos do VSF pode ser atribuída às antocianinas ou também componentes fenólicos incolores ou amarelos, tais como os estilbenos, as flavonas e os flavan-3-ois. De acordo com Lins (2014), a qualidade dos compostos bioativos do vinho esta ligada à qualidade da uva, e a uva é uma das maiores fontes de compostos fenólicos, principalmente os flavonoides (antocianinas, flavanóis e flavonóis), os estilbenos (resveratrol), os ácidos fenólicos (derivados dos ácidos cinâmicos e benzóicos) e uma larga variedade de taninos. Compostos estes que além de contribuírem para $\mathrm{O}$ flavor e a cor dos vinhos possuem propriedades antioxidantes. 
Tabela 2 - Variáveis meteorológicas (precipitação pluvial, temperaturas do ar - mínima, máxima, e média - e amplitude térmica) obtidas das estações meteorológicas localizadas em Uruguaiana, Bagé, Caxias do Sul, Bento Gonçalves, Petrolina e São Joaquim, de outubro de 2008 a março de 2009.

\begin{tabular}{|c|c|c|c|c|c|c|c|}
\hline \multicolumn{8}{|c|}{ Precipitaçào Mensal (mm) } \\
\hline Periodo & Uruguaiana & Bagé & Caxias do Sul & Bento Gonçalves & Petrolina & Petrolina* & Săo Joaquim \\
\hline Outubro de 20008 & 236.3 & 97.9 & 267.3 & 309.6 & 00 & 902 & 3828 \\
\hline Novembro de 2008 & 231. & 51.9 & 1041 & 70.3 & 0.0 & 55.3 & 236.3 \\
\hline Dezembro de 2008 & 21.9 & 73.8 & 101.6 & 85.8 & 63.1 & 711 & 92.6 \\
\hline Janeiro de 2009 & 123.9 & 98,3 & 226.7 & 269.6 & 5.4 & 3.4 & 1922 \\
\hline Fevereiro de 2009 & 146.5 & 156.1 & 148.7 & 144.5 & 121.4 & 34 & 111.5 \\
\hline Março de 2009 & 139.0 & 45.2 & 62.4 & 90.6 & 116.4 & 0.0 & 93.1 \\
\hline \multicolumn{8}{|c|}{ Temperatura Minima Mensal $\left({ }^{\circ} \mathrm{C}\right)$} \\
\hline Periodo & Uruguaiana & Bagé & Caxias do Sul & Bento Gonçalves & Petrolina & Petrolina ${ }^{x}$ & Săo Joaquim \\
\hline Outubro de 2008 & 14,9 & 12.5 & 11.7 & 13.1 & 210 & 23.5 & 10.2 \\
\hline Novembro de 2008 & 17.0 & 15.2 & 12.9 & 14.7 & 224 & 22.6 & 109 \\
\hline Dezembro de 2008 & 18.6 & 16.0 & 13.7 & 15.6 & 22.3 & 214 & 11.2 \\
\hline Janeiro de 2009 & 18.4 & 16.8 & 143 & 16.1 & 229 & 20.9 & 12,7 \\
\hline Fevereiro de 2009 & 192 & 17.7 & 157 & 17.8 & 221 & 21.1 & 143 \\
\hline Março de 2009 & 17.4 & 16.9 & 14.9 & 17.1 & 23.9 & 22.6 & 13.7 \\
\hline \multicolumn{8}{|c|}{ Temperatura Máxima Densal $\left({ }^{\circ} \mathrm{C}\right)$} \\
\hline Periodo & Uruguaiana & Bagé & Caxias do Sul & Bento Gonçalves & Petrolina & Petrolina ${ }^{*}$ & Săo Joaquim \\
\hline Outubro de 2008 & 247 & 221 & 20.4 & 21.2 & 343 & 30.8 & 17.2 \\
\hline Novembro de 2008 & 30.4 & 27.5 & 233 & 25 & 355 & 290 & 18.5 \\
\hline Dezembro de 2008 & 32.1 & 27.9 & 248 & 262 & 33.4 & 29.5 & 21,3 \\
\hline Janeiro de 2009 & 31.5 & 29,1 & 24.1 & 25,7 & 34.0 & 302 & 21.0 \\
\hline Fevereiro de 2009 & 30.9 & 29.1 & 26.6 & 26.6 & 32.2 & 312 & 22.7 \\
\hline Março de 2009 & 30,1 & 27.4 & 25.0 & 26.2 & 33.0 & 342 & 22.4 \\
\hline \multicolumn{8}{|c|}{ Temperatura Média Mensal $\left({ }^{\circ} \mathrm{C}\right)$} \\
\hline Periodo & Uruguaiana & Bagé & Caxias do Sul & Bento Gonçalves & Petrolina & Petrolinax & Säo Joaquim \\
\hline Outubro de 2008 & 19.4 & 17.6 & 16.0 & 16.6 & 27.6 & 27,1 & 13.0 \\
\hline Novembro de 2008 & 23.7 & 21.4 & 18.1 & 19.4 & 28.7 & 25.9 & 140 \\
\hline Dezembro de 2008 & 25.4 & $22: 1$ & 19.2 & 20.3 & 27.9 & 25.5 & 15.3 \\
\hline Janeiro de 2009 & 25.0 & 22.6 & 19.2 & 20.4 & 28.5 & 25.6 & 15.9 \\
\hline Fevereiro de 2009 & 25,1 & 23.2 & 20.6 & 21.7 & 27.1 & 26.1 & 176 \\
\hline Março de 2009 & 23.8 & 222 & 19.8 & 21.0 & 28.5 & 28.3 & 16.9 \\
\hline \multicolumn{8}{|c|}{ Amplitude Térmica Mensal $\left({ }^{\circ} \mathrm{C}\right)$} \\
\hline Periodo & Uruguaiana & Bagé & Caxias do Sul & Bento Gonçalves & Petrolina & Petrolina & Säo Joaquim \\
\hline Outubro de 2008 & 10.5 & 9.6 & 8.7 & 8.1 1 & 13.3 & 7.3 & 7.0 \\
\hline Novembro de 2008 & 135 & 12.4 & 10.4 & 10.3 & 13.1 & 6.4 & 7.6 \\
\hline Dezembro de 2008 & 13.5 & 11.9 & 11.1 & 10.6 & 11.1 & 8.1 & 10.1 \\
\hline Janeiro de 2009 & 130 & 12.3 & 98 & 9.6 & 11,1 & 9.3 & 8.3 \\
\hline Fevereiro de 2009 & 11.8 & 114 & 97 & 8.8 & 10.1 & 10.1 & 84 \\
\hline Março de 2009 & 12.7 & 10.6 & 10.2 & 9,1 & 9.1 & 11.6 & 8.7 \\
\hline
\end{tabular}

Fonte: Dados de Uruguaiana e Caxias do Sul (CemetRS/FEPAGRO); dados de Bagé, Petrolina, Petrolina* e São Joaquim (INMET); dados de Bento Gonçalves (EMBRAPA). 


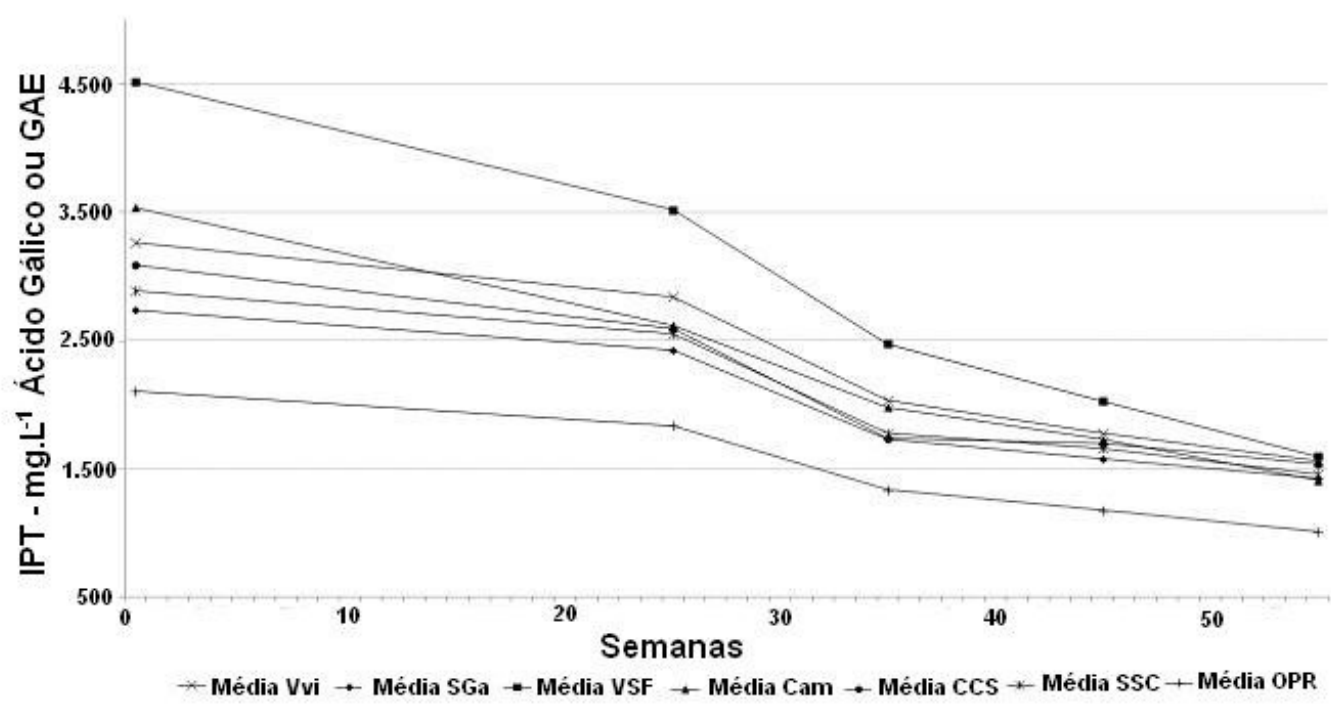

Figura 1 - Evolução dos compostos fenólicos (IPT) de vinhos tintos procedentes de diferentes regiões do Brasil no período de zero a 55 semanas. Dados médios para as amostras das regiões Vale dos Vinhedos (Vvi), Serra Gaúcha (SGa), Vale do Rio São Francisco (VSF), Campanha Gaúcha (Cam), Campos de Cima da Serra (CCS), Serra de Santa Catarina (SSC) e Oeste do Paraná (OPR).

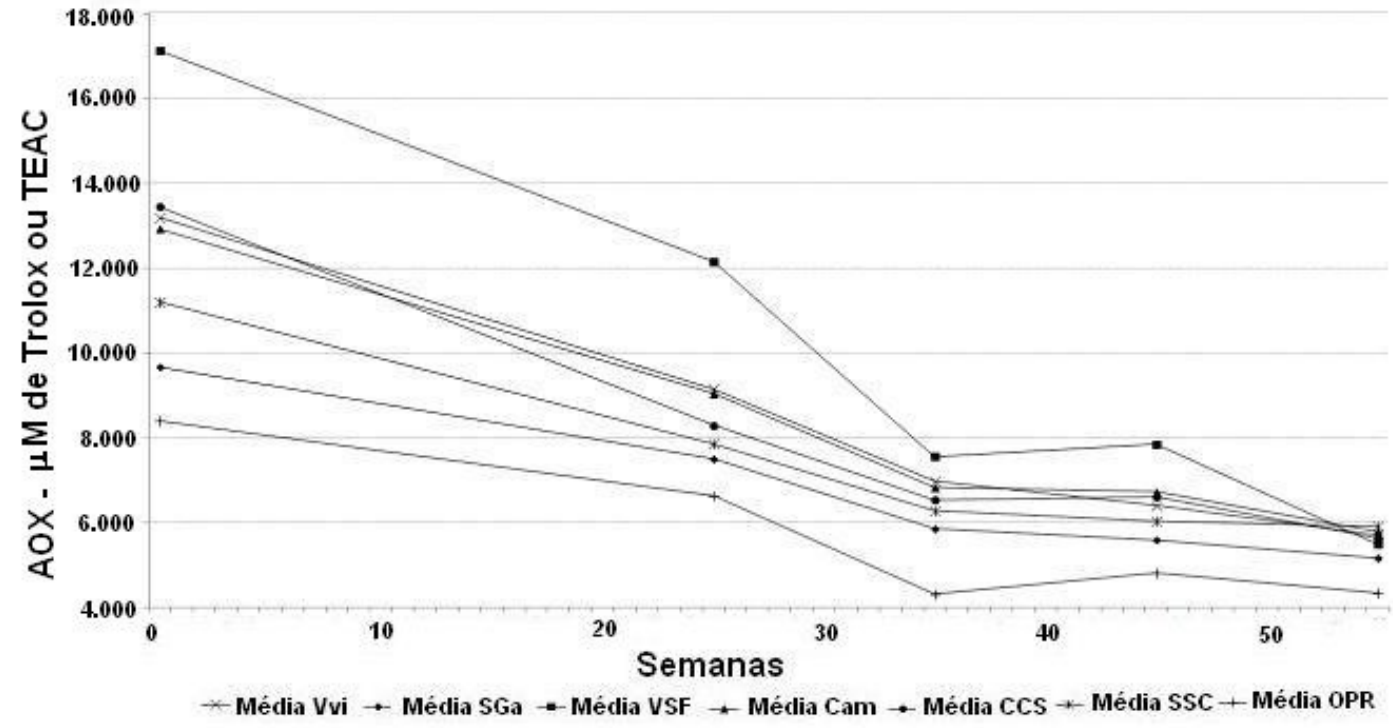

Figura 2 - Evolução da atividade antioxidante (AOX) de vinhos tintos procedentes de diferentes regiões do Brasil no período de zero a 55 semanas. Dados médios para as amostras das regiões Vale dos Vinhedos (Vvi), Serra Gaúcha (SGa), Vale do Rio São Francisco (VSF), Campanha Gaúcha (Cam), Campos de Cima da Serra (CCS), Serra de Santa Catarina (SSC) e Oeste do Paraná (OPR).

As condições de estresse hídrico a que estão submetidas as videiras no VSF, associadas à maior disponibilidade de radiação solar (com pouca variação interanual) podem propiciar uma maior síntese de ácido abcísico (ABA) e de flavonoides. Berli (2011) encontrou alterações no perfil de antocianinas e de outros compostos fenólicos em uvas Merlot tratadas com irradiação UV e ABA em máximas concentrações. O estresse hídrico, quando bem manejado, 
naturalmente leva a uma concentração de ABA na planta, especialmente quando for aplicada a estratégia de manter parcialmente seca a zona de raiz (STOLL et al., 2000).

Analisando-se os dados de insolação da região de Bento Gonçalves, observase que os períodos mais determinantes para a síntese de compostos fenólicos, os quais acontecem na fase final do ciclo reprodutivo (do início da troca de cor ao amadurecimento e colheita), não coincidem com os maiores valores de insolação do período analisado, os quais ocorreram antes desse período crítico mencionado. Assim sendo, os perfis temporais de IPT e AOX dos vinhos provenientes da região de Bento Gonçalves para a safra 2008/2009 são reflexo de uma condição menos favorecida, do ponto de vista da variável climática insolação. Este aspecto certamente influenciou a síntese e o acúmulo de compostos na uva, tendo reflexo nos perfis temporais de IPT e AOX (PEDRO JR e SENTELHAS, 2003).

Para a coloração adequada das bagas e para um desejável acúmulo se sólidos solúveis, são necessárias entre 1200 e 1400 horas de sol durante todo o ciclo vegetativo da videira, desde a saída da dormência até a colheita (PEDRO JR e SENTELHAS, 2003). As regiões que mais oferecem essa disponibilidade climática são VSF e Cam, justamente as regiões que apresentaram os mais altos valores iniciais de IPT e, também elevados valores para AOX, demonstrando que os vinhos elaborados com uvas daquelas regiões, são resultado de uma condição de síntese e acúmulo de polifenóis favorecida pela disponibilidade de horas de sol.

Regiões subtropicais são capazes de compensar em termos climáticos sua condição de baixa latitude quando se encontram situadas em elevada altitude. Fatores como a amplitude térmica e a variação no fotoperíodo são intensificadas, portanto, em relevos de Serra (PEDRO JR e SENTELHAS, 2003). Dessa maneira, as regiões serranas VVi, CCS, SSC, SGa podem atribuir em parte o seu IPT e AOX a essa condição climática, importante para um melhor desempenho da cultura da uva.

Os valores mais baixos, em todos os tempos, para ambas as análises (IPT e AOX), ocorridos em OPR podem indicar uma série de possibilidades, que vão desde que a combinação das condições ambientais do ano agrícola 2008/2009 não favoreceu a safra da uva vinífera, independentemente do manejo adotado, até que, desempenhos mais elevados, em termos de IPT e AOX, as condições ambientais existentes, recorrentes a cada ano, naquele perímetro de produção limitem os desempenhos da videira vinífera, em termos de IPT e AOX, fato esse que acarretaria, em uma sequência de anos analisados, em valores semelhantes aos encontrados nesse trabalho. Estudos com variedades mais adaptadas àquele ambiente podem contribuir para um desempenho mais interessante do ponto de vista de IPT e AOX para OPR.

Em relação às amostras provenientes do VSF, a baixa amplitude térmica diária distribuída uniformemente ao longo do ano (Tabela 2), indica que as condições foram insuficientes para a síntese de pigmentos. Entretanto, considerando-se que os maiores valores de insolação (Figura 3) e os menores volumes de precipitação pluvial (Tabela 2) ocorrem naquela região, a síntese de polifenóis pode ter sido favorecida. Pereira et al. (2009) destaca que a maturação rápida da uva provocada por altas temperaturas do ar pode gerar um descompasso entre a maturação tecnológica e a maturação fenólica. Os vinhos 
resultantes apresentam baixa estabilidade de compostos de cor, altos $\mathrm{pH}$ 's, alta sensibilidade à oxidação, taninos duros e adstringentes, o que dá a esse tipo de produto baixo potencial para o armazenamento.

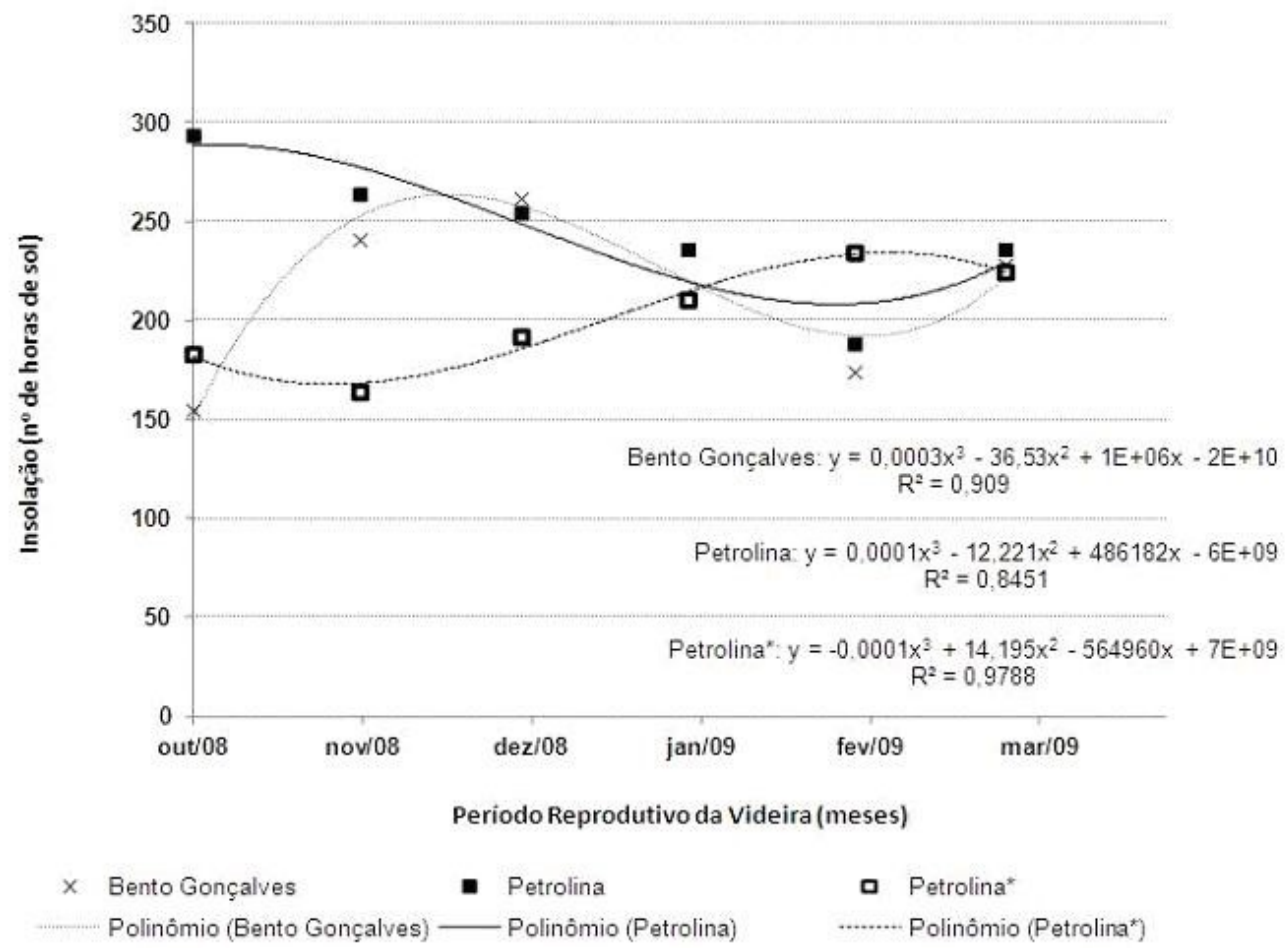

Figura 3 - Insolação mensal (número de horas de sol) durante o período reprodutivo da videira (outubro de 2008 a março de 2009) das estações meteorológicas localizadas em Bento Gonçalves-RS e Petrolina-PE. Os dados Petrolina* correspondem ao período de Abril de 2009 a Setembro de 2009, por considerar a possibilidade de que o ciclo reprodutivo da planta possa ter acontecido neste período.

Em relação ao IPT a AOX encontrados neste trabalho, as variedades Merlot $\mathrm{e}$ Cabernet sauvignon não apresentaram diferenças significativas pelo teste $t$ de student para um $\alpha$ de 0,1 . Spinelli et al. (2006) encontraram maiores teores de trans-resveratrol e estilbenos totais superiores em vinhos Merlot quando comparados aos vinhos tannat e Cabernet sauvignon. Dias (2009) também encontrou teores mais elevados de resveratrol na variedade Merlot, o que pode culminar com IPT e AOX diferentes em função da variedade, já que, qualitativamente, as uvas diferem entre si e o tipo de composto fenólico predominante influencia a AOX de vinhos.

A análise de correlação entre os dados confirma a associação linear entre IPT e AOX. Para os tempos avaliados, e segundo a classificação de Hair (2004), os dados apresentaram os seguintes valores de correlação: tzero $=0,96 ; \mathrm{t} 25=0,96$; $\mathrm{t} 35=0,92 ; \mathrm{t} 45=0,95$ e $\mathrm{t} 55=0.83$. Esse resultado era esperado em função da capacidade antioxidante exercida pelos compostos fenólicos.

Nos períodos analisados, as maiores quedas nos valores de IPT e AOX são observadas entre t25 e t35. Esse intervalo de tempo coincide com a maior queda 
de temperatura do ar no local de armazenamento dos vinhos, ocorrida entre março de 2011 e julho de 2011 (Figura 4) e com o mais longo período, em dias contínuos, nos quais a umidade relativa do ar permaneceu abaixo de $65 \%$ (Figura 5). A temperatura do ar interfere na estabilidade de vinhos, e o efeito mais pronunciado desta influência ocorreu no final do verão. Neste período, a diminuição da temperatura do ar pode ter favorecido a precipitação dos compostos fenólicos e, consequentemente, a diminuição do IPT e da AOX dos vinhos.

O perfil de evolução temporal dos grupos, independentemente dos seus valores iniciais de IPT e AOX, tende a diminuir as diferenças entre si. Ao serem igualadas condições de temperatura, umidade e estocagem, as variações agroclimáticas e enológicas que definiram diferenças, em termos de IPT e AOX, aos vinhos provenientes de diferentes regiões brasileiras, foram reduzidas. As condições relativas ao armazenamento (temperatura, umidade e tipo de frasco) podem ter determinado o perfil temporal de evolução, minimizando em 55 semanas as diferenças finais entre os vinhos analisados, em termos de IPT e AOX.

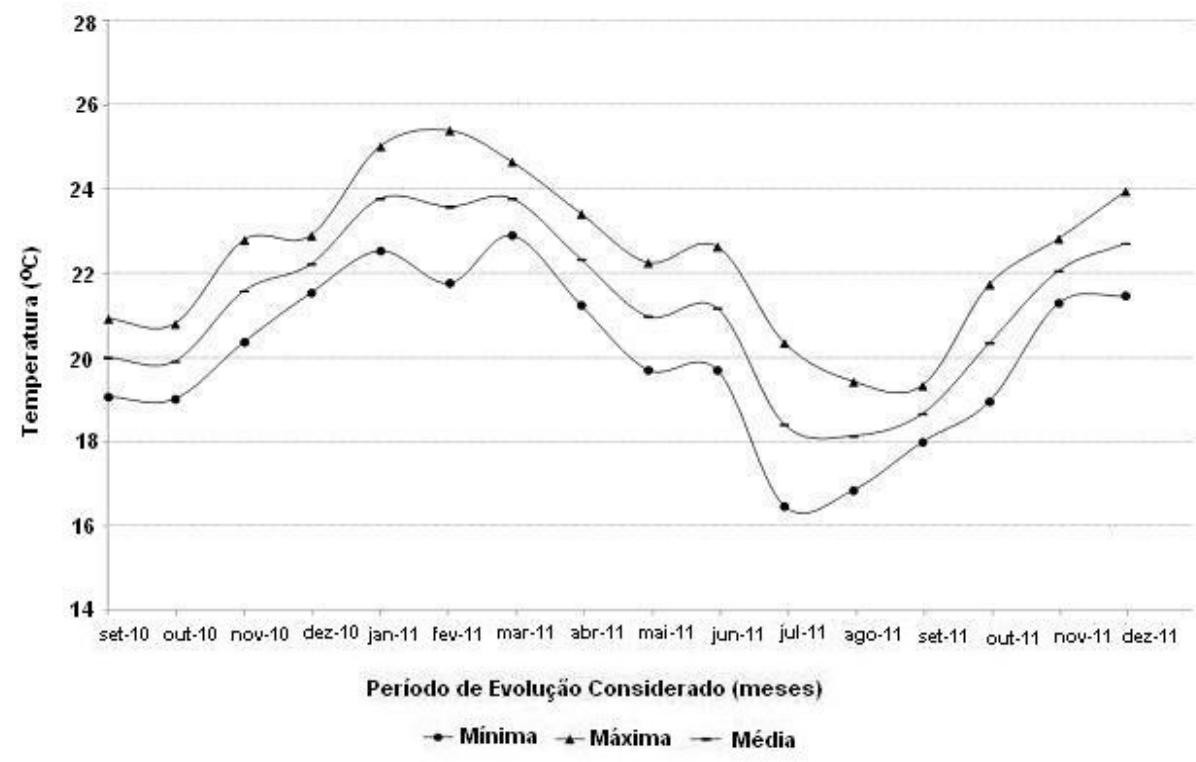

Figura 4 - Variação de temperatura ambiente no local de estocagem dos vinhos durante o período de análise. 


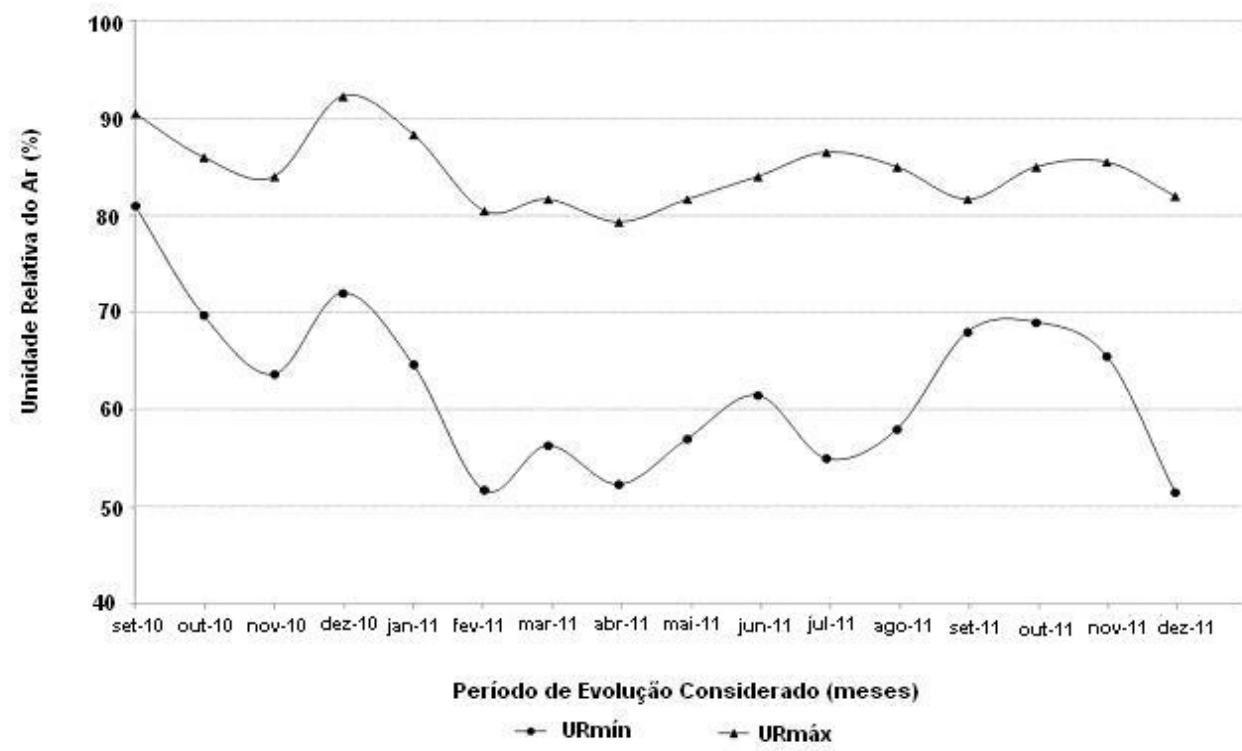

Figura 5 - Variação da umidade ambiente no local de estocagem dos vinhos durante o período de análise.

\section{CONCLUSÃO}

De maneira geral, todos os vinhos analisados apresentaram qualidade satisfatória nos índices de polifenóis totais e atividade antioxidante, com a redução do IPT e AOX ao longo do período de armazenamento, independentemente da região fisiográfica de procedência dos vinhos.

Os dados obtidos neste trabalho podem ser utilizados como base para avaliar a qualidade antioxidante dos vinhos em relação à potencialidade das regiões produtoras. O perfil evolutivo temporal de IPT e AOX pode ser uma informação a se levar em consideração na hora de se definir os perímetros de produção, baseados na tipicidade e autenticidade dos vinhos. Sendo que, os vinhos de regiões brasileiras apresentam condições climáticas diferenciadas, e conseguem ser caracterizados, em termos de IPT e AOX, de forma distinta. 


\title{
Brazilian Journal of Food Research
}

ISSN: 2448-3184

\section{Temporal Evolution of Phenolic Compounds and Antioxidant Activity of Brazilian Red Wines}

\begin{abstract}
Based on the study of 90 wine samples from seven different Brazilian regions, the total polyphenol content and antioxidant activity indicated a distinct evolution. The variables studied showed strong and very strong correlation during 55 week. The region of Vale do São Francisco exhibited highest polyphenol content in all analysis times and higher antioxidant activity up to 45 weeks. The region of Oeste do Paraná had the lowest values for both parameters in all evaluated times. The temporal evolution of phenolic compounds and antioxidant activity showed that the differences between wines from different regions of origin decrease with storage. Characteristics indicated by weather variables may have favored the initial differences, while at the end of 55 weeks the storage conditions approached the most of the groups.
\end{abstract}

KEYWORDS: Polyphenols; Antioxidant activity; Wine characterization; Brazilian terroir. 


\section{REFERÊNCIAS}

BARON-MENGUY, C.; BOCQUET, A.; GUIHOT, A. L.; CHAPPARD, D.; AMIOT, M. J.; ANDRIANTSITOHAINA, R.; LOUFRANI, L.; HENRION, D. Effects of red wine polyphenols on postischemic neovascularization model in rats: low doses are proangiogenic, high doses anti-angiogenic. The FASEB Journal, v. 21, n. 13, p. 3511-21, 2007.

BAVARESCO, L.; GATTI, M.; PEZZUTTO, S.; FREGONI, M.; MATTIVI, F. Effect of leaf removal on grape yield, berry composition and stilbene concentration. American Journal of Enology and Viticulture, v. 59, n. 3, p. 292-298, 2008.

BERLI, F.; FANZONE, M.; PICCOLI, P.; BOTTINI, R. Solar UV-B and ABA are involved in phenol metabolism of Vitis vinifera $L$. increasing biosynthesis of berry skin polyphenols. Journal of Agricultural and Food Chemistry, v. 59, v. 9, p. 4874-4884, 2011.

BRAND-WILLIAMS, W.; CUVELIER, M. E.; BERSET, C. Use of a Free Radical Method to Evaluate Antioxidant Activity. Lebensmittel-Wissenschaft und Technologie Food Science and Technology, v. 28, n. 1, p. 25-30, 1995.

CANO-LÓPEZ, M.; PARDO-MÍNGUEZ, F.; LÓPEZ-ROCA, J.M.; GÓMEZ-PLAZA, E. Chromatic characteristics and anthocyanin profile of a micro-oxygenated red wine after oak or bottle maturation. European Food Research and Technology, v. 225, n. 1, p. 127-132, 2007.

CELOTTI, E.; FERRARINI, R.; ZIRONI, R.; CONTE, L. S. Resveratrol content of some wines obtained from dried Valpolicella grapes: Recioto and Amarone. Journal of Chromatography A, v. 730, n. 1-2, p. 47-52, 1996.

DIAS, J. F. Determinação dos conteúdos de resveratrol em vinhos tintos de duas regiões brasileiras. 2009. 100 f. Dissertação (Mestrado em Ciência de Alimentos), Universidade Federal do Rio de janeiro, 2009.

FRANKEL, E. N.; WATERHOUSE, A. L.; TEISSEDRE, P. L. Principal phenolic phytochemicals in selectes California Wines and their antioxidant activity in inhibiting oxidation of human low-density lipoproteins. Journal of Agriculture and Food Chemistry, v. 43, n. 4, p. 890-894,1995.

GIOVANINNI, E.; MANFROI, V. Viticultura e enologia - elaboração de grandes vinhos nos Terroirs Brasileiros. IFRS - Instituto Federal de Educação, Ciência e Tecnologia do Rio Grande do Sul, 344 p., 2009. 
HINNES, L. M.; STAMPFER, M. J.; MA, J.; GAZIANO, J. M.; RIDKER, P. M.; HANKINSON, S. E.; SACKS, F.; RIMM, E. B.; HUNTER, D. J. Genetic variation in alcohol dehydrogenase and the beneficial effect of moderate alcohol comsumption on myocardial infarction. The New England Journal of Medicine, v. 344, n. 8, p. 549-555, 2001.

HORNSEY, I. The chemistry and biology of winemaking. The Royal Society of Chemistry, Cambridge, UK, 2007.

KENNEDY, J. A.; MATTHEWS, M. A.; WATERHOUSE, A. L. Effect of maturity and vine water status on grape skin and wine flavonoids. American Journal of Enology and Viticulture, v. 53, n. 4, p. 268-274, 2002.

KLATSKY, A.L.; ARMSTRONG, M.A.; FRIEDMAN, G.D. Alcohol and mortality. Annals of Internal Medicine, v. 117, n. 8, p. 646-654, 1992.

KOLOUCHOVÁ-HAZLÍKOVÁ, I.; MELZOCH, K.; FILIP, V.; SMIDRKAL, J. Rapid method for resveratrol determination by HPLC with electrochemical and UV detections in wines. Food Chemistry, v. 87, n. 1, p. 151-158, 2004.

KRAMER, M. Denominação e autenticidade. In: Os sentidos do vinho. Conrad editora do Brasil. p. 67-85, São Paulo, 2007.

LANDRAUT, N.; POUCHERET, P; RAVEL, P.; GASC, F.; CROS, G.; TEISSEDRE, P.L. Antioxidant capacities and phenolics levels of French wines from different varieties and vintages. Journal of Agriculture and Food Chemistry, v. 49, n. 7, p. 3341-3348, 2001.

LINS A. R, GILIANI V. S. Qualidade Fenólica e Atividade Antioxidante de Vinhos Tintos Produzidos no Estado do Paraná. Revista Brasileira de Produtos Agroindustriais, v.16, n.1, p.69-76, 2014

LONGNECKER, M. P. Alcoholic beverage consumption in relation to risk of breast cancer: meta-analysis and review. Cancer Causes Control, v. 5, n. 1, p. 73-82, 1994.

MAMEDE, M. E. O.; PASTORE, G. M. Compostos fenólicos do vinho: estrutura e ação antioxidante. Boletim CEPPA, v. 22, n. 2, p. 233-252, 2004.

MATEUS, N.; PROENÇA, S.; RIBEIRO, P.; MACHADO, J. M.; FREITAS, V. de. Grape and wine polyphenolic composition of red Vitis vinifera varieties concerning vineyard altitude. Ciencia y Tecnología Alimentaria, v. 3, n. 2, p. 102-110, 2001. 
MATTIVI, F.; GUZZON, R.; VRHOVSEK, U.; STEFANINI, M.; VELASCO, R. Metabolite profiling of grape: flavonols and anthocyanins. Journal of Agriculture and Food Chemistry, v. 53, n. 20, p. 7692-7702, 2006.

MC'ELDUFF, P.; DOBSON, A. J. How alcohol and how often? Population based case-control study of alcohol consumption and risk of a major coronary event. British Medical Journal, v. 314, p. 1159-1164, 1997.

PEREIRA, G. E.; GUERRA, C. C.; MANFROI, L. Vitivinicultura e Enologia. In: SOARES, J. M.; LEÃO, P. C. S. A Vitivinicultura no Semiárido brasileiro. Petrolina: EMBRAPA Semi-Árido, 2009. 756 p.

RE, R.; PELLEGRINI, N.; PROTEGGENTE, A.; PANALA, A.; YANG, M.; RICE-EVANS, C. Antioxidant activity applying an improved ABTS radical cation decolorization assay. Free Radical Biology and Medicine, v. 26, n.9-10, p. 1231-1237, 1999.

RICE-EVANS, C.A.; MILLER, N.J. Antioxidant activities of flavonoids as bioactive components of food. Biochemistry Society Transactions, v.24, n. 3, p. 790-795, 1996.

RIVERO-PÉREZ, M.D.; MUÑIZ, P.; GONZÁLEZ-SANJOSÉ. Antioxidant profile of red wines evaluated by total antioxidant capacity, scavenger capacity, and biomarkers of oxidative stress methodologies. Journal of Agriculture and Food Chemistry, v. 55, n. 14, p. 5476-5483, 2007.

SCANDALIOS, J.G. Oxygen stress and superoxide dismutases. Plant Physiology, v. 101, n. 1, p. 7-12, 1993.

SINGLETON, V. L.; ESAU, P. Phenolic Substances in grape and wine, and their significance. Advances in Food Research Supplement, v. 1, n. 1, p. 1-261, 1969.

SPINELLI, F. R.; VANDERLINDE, R.; DUTRA, S. V.; PEDRUZZI, I.; MARCON, A. R.; BALARDIN, P. B. Teores de resveratrol em vinhos do Rio Grande do Sul. In: REUNIÃO ANUAL DA SOCIEDADE BRASILEIRA PARA O PROGRESSO DA CIÊNCIA, 58, 2006. Florianópolis, SC. Anais... Florianópolis, SBPC, 2006.

STOLL, M.; LOVEYS, B.; DRY, P. Hormonal changes induced by partial rootzone drying of irrigated grapevine. Journal of Experimental Botany, v. 51, n. 350, p. 1627-1634, 2000. antioxidative enzymes and total anthocyanins in natural populations of Iris pumilla L. Journal of Plant Ecology, v. 3, n. 1, p. 59-69, 2010. 
WANG, H; COA, G.; PRIOR, R. L. Oxygen radicals absorbing capacity of anthocyanins. Journal of Agriculture Food Chemestry, v. 45, n. 2, p. 304-309, 1997.

WATERHOUSE, A. Determination of Total Phenolic. In: Current Protocols in Food Analytical Chemistry. Wiley, 2002, I1.1.1-I1.1.8.

Recebido: 08 jan. 2016.

Aprovado: 26 set. 2016

DOI: $10.14685 /$ rebrapa.v8n1.3673

Como citar:

DULLIUS, M. et al. Evolução temporal de compostos fenólicos e atividade antioxidante de vinhos tintos brasileiros. Brazilian Journal of Food Research, Campo Mourão, v. 8, n.1, p. 38-55, jan./mar. 2017.

Disponivel em: https://periodicos.utfpr.edu.br/rebrapa

Correspondência:

Alessandro Rios

Universidade Federal do Rio Grande do Sul, Brasil.

Direito autoral: Este artigo está licenciado sob os termos da Licença Creative Commons-Atribuição 4.0

Internacional.

\section{(c) (1)}

